\title{
Maroc - Enquêtes internationales en Éducation :
}

\section{Quelles leçons ?}

\section{Mohamed STITOU Association AMAQUEN}

Cet article est consacré à la participation du Système d'Éducation et de Formation (SÉF) marocain aux enquêtes internationales en Éducation, en l'occurrence TIMSS2011 et PIRLS2011. Enquêtes auxquelles notre pays participe respectivement depuis 1995 et 2001 dans le but de comparer ses politiques et ses pratiques éducatives, à celles des autres participants. La liste des participants regroupe des pays de catégories socio-économiques très diverses. Nous essayons donc, à travers cet article, de relater les tenants et les aboutissants de cette participation, qui s'opère au nom de Notre pays, le MAROC.

Et, dans le souci de permettre aux lectrices et lecteurs d'appréhender, le plus profondément possible, ce sujet, qui trouve sans nul doute intérêt chez tous les citoyens, notre lecture commentée des résultats consignés dans les rapports des études TIMSS2011 et PIRLS2011 parus le 11 décembre 2012, sera précédée d'un bref historique, d'une définition du processus et du contexte dans lequel ces enquêtes s'opèrent tant en amont qu'en aval.

\section{Un bref historique}

Depuis les années quarante, soit au lendemain de la seconde Guerre mondiale, l'analyse des performances scolaires se basait uniquement sur des indicateurs quantitatifs. Approche qui rimait parfaitement avec la nature des contextes de l'époque. En effet les préoccupations qui prévalaient, chez les décideurs nationaux à travers le monde, étaient centrées sur les problématiques liées à l'accès à l'éducation et à la continuité des flux scolaires. En d'autres termes, le souci majeur était de répondre aux questions de

Organisées toutes les deux par l'IEA (International Association for the Evaluation of Educational Achievement), les enquêtes PIRLS et TIMSS, dont la périodicité est différente, ont coïncidé en 2011, ce qui a permis en quatrième année primaire, le niveau commun à ces deux enquêtes, de recueillir pour la même cohorte, des données en trois disciplines, les mathématiques, les sciences et la lecture. Ce qui, de facto, accorde aux pays participants la précieuse possibilité d'évaluer la performance globale de ces mêmes élèves.

Les scores sont établis sur une échelle de 1000 points avec une moyenne servant de repère de comparaison, fixée à 500 points.

Notons qu'à l'issu de chaque session et pour chacune des deux études l'IEA publie en volumes séparés, le guide utilisateur de la base de données, le rapport général regroupant résultats et analyses, et l'encyclopédie présentant les systèmes éducatifs de tous les pays participants.

comment améliorer les taux de scolarisation, de réduire celui de l'abandon, et d'améliorer la transition entre cycles d'enseignement. 
Il fallait donc attendre l'aube des années soixante pour que la qualité des apprentissages, et non plus seulement le nombre d'enfants scolarisés ou d'années de scolarisation dispensées, se révélait comme facteur déterminant dans la mesure de la pertinence des politiques éducatives et de l'efficacité des pratiques éducatives. La mesure et l'évaluation de la qualité des apprentissages des élèves se dressaient en défi à relever. À cet effet, un petit groupe de spécialistes en évaluation s'est réuni en 1958 à l'UIE (Unesco Institute of

Organisée en 2001, 2006 et 2011, PIRLS (Progress in International Reading Literacy Study) est un programme d'étude comparée (benchmarking) portant sur les compétences en lecture et compréhension chez les élèves de la quatrième année de l'enseignement élémentaire (cycle de l'enseignement primaire chez nous). Elle est organisée tous les cinq ans. Elle vise l'évaluation des acquis en tenant compte de l'environnement dans lequel se réalisent les apprentissages, notamment le curriculum, les ressources dont dispose l'école, les effectifs en classe, la formation des enseignants ... ainsi que les conditions qu'offre la famille comme les ressources matérielles, le niveau d'instruction des parents et le niveau socioéconomique de la famille.

l'enquête PISA.

Il est, sans doute, important de noter qu'en plus de l'évaluation directe des acquis des élèves à travers les tests disciplinaires, ces enquêtes

La participation à PIRLS coûte à chaque pays 225 mille euro environ, et celle de TIMSS 180 mille euro. Si un pays participe aux deux études, sa contribution est de l'ordre de 300 mille euro.

s'articulent sur le principe de la collecte de statistiques et des données au niveau des pays concernés. Elles visent la mise en évidence des facteurs déterminants des performances des Organisée en 1999, 2003, 2007 et 2011, TIMSS (Trends in International Mathematics and Science Study) est un programme d'étude comparée (benchmarking) portant sur les compétences des élèves en mathématiques et en sciences. Cette étude, s'opère en deux niveaux scolaires, à savoir la quatrième année de l'enseignement élémentaire (cycle de l'enseignement primaire chez nous) et l'avant dernière année de l'enseignement fondamental (deuxième année cycle secondaire collégial au Maroc). Elle est organisée tous les quatre ans.

Education) à Hambourg pour concevoir un dispositif permettant d'évaluer la qualité des apprentissages des élèves dans différents pays à travers un test de connaissances

international unique et standardisé. Cette nouvelle logique allait donner un nouvel essor aux enquêtes internationales. Aux indicateurs quantitatifs jusqu'alors mobilisés, on commence à opposer une nouvelle vision qualitative. Depuis, l'amélioration continue de ces enquêtes n'a cessé d'être observée que ce soit au niveau de l'agence non gouvernementale américaine l'IEA auteure des enquêtes PIRLS et TIMSS ou encore l'organisation intergouvernementale, l'OCDE auteure de

En termes de statistique, PIRLS $2011 \mathrm{a}$ vu la participation des élèves du primaire à la quatrième année scolaire de 45 pays. En moyenne 4000 élèves ont représenté chaque pays, soit un échantillon de 175 écoles en moyenne. Le nombre total de participants était de 325000 élèves.

Alors qu'en TIMSS 2011 se sont 63 pays qui ont participé, répartis entre 52 pays au niveau de la quatrième année primaire et 45 au niveau de la deuxième année du collège. Les élèves ayant participé sont de l'ordre de 600 mille élèves.

systèmes éducatifs. Pour cela, elles mettent en relation les performances des organisations scolaires avec à la fois les caractéristiques socio-économiques des publics scolaires accueillis, 
et les conditions d'enseignement analysées à travers le double prisme des politiques éducatives et des pratiques pédagogiques au niveau des classes et des établissements scolaires. Par conséquent, leurs résultats s'avèrent aussi prisés qu'utiles dans l'élaboration de stratégies d'amélioration de la part des responsables politiques, des spécialistes du domaine de l'Éducation et des chercheurs.

\section{Participation du Maroc}

Le Maroc a été représenté par un échantillon de 7841 élèves issus de 273 écoles primaires et de 8986 élèves représentant 333 collèges.

La participation du Maroc à ces deux enquêtes, en l'occurrence PIRLS2011 et TIMSS2011, revêt un aspect particulier. En effet, elle survient à une date significative à plus d'un égard. D'une part, c'est une année qui vient après la clôture de la décennie déclarée par notre pays, en tant que décennie de l'Éducation et la Formation, une sorte d'évaluation de l'adéquation des recommandations de la Charte Nationale de l'Éducation et de Formation et de la manière avec laquelle celles-ci ont été mises en œuvre. Et d'autre part, cette date qui correspond à la veille de l'achèvement de l'ambitieux Programme d'Urgence (2009-2912). Ce dernier, qui s'est apparemment donné les moyens financiers nécessaires, sans pour autant pouvoir tenir ses promesses de donner un nouvel élan à la réforme entamée en 2000, soit le second souffle. C'est une occasion en or, pour faire une lecture approfondie des maux chroniques qui nuisent à l'efficacité et l'efficience de notre système éducatif.

\section{Scores des élèves Marocains}

Quant aux résultats des élèves marocains au niveau de la quatrième année du primaire en mathématiques et sciences, et à l'instar de l'édition 2007, ils sont restés loin en dessous de la moyenne internationale. Ils ont obtenu 264 points dans les sciences avec un écart de 236 points par rapport à la moyenne internationale fixée à 500 points. En mathématiques leur score n'a guère dépassé les 335 points, soit 165 points en dessous de la moyenne internationale. La tendance est à la baisse par rapport aux résultats de nos élèves en 2007. En effet, les scores obtenus en 2011 sont inférieurs à ceux obtenus en 2007 : en mathématiques le recul est de six points, alors qu'en sciences ce recul atteint les 33 points.

En ce qui concerne le niveau de la deuxième année collégiale le Maroc n'a pas fait mieux. Nos élèves ont réalisé en sciences un score de 376 points soit 124 points au-dessous de la moyenne internationale, et en mathématiques un score de 371 points, 129 points de moins que la moyenne internationale. Toujours, en comparaison avec les scores enregistrés en 2007, les élèves marocains ont régressé de dix points en mathématiques, et de 26 points en sciences.

S'agissant du classement général, au PIRLS, le Maroc a occupé la dernière place, devancé de 81 points par Oman classé $41^{\text {ème }}$; alors qu'à TIMSS, en mathématiques et sciences au niveau de la $4^{\text {ème }}$ année du primaire, le Maroc a occupé l'avant dernière place en cédant de justesse la dernière au Yémen. En fin, à TIMSS, au niveau de la $2^{\text {ème }}$ année du collège, le Maroc a devancé en mathématiques deux pays Oman et le Ghana ; et en sciences seulement le Ghana. 
En ce qui concerne l'étude PIRLS2011, qui mesure la performance des apprenants en lecture, le Maroc est passé du rang avant-dernier qu'il a occupé en 2006, avec 323 points et un écart de -177 points par rapport au niveau moyen international, au dernier rang en 2011 avec un total de 310 points et l'écart de -190 points sur la moyenne internationale. Résultat qui l'a classé derrière plusieurs pays arabes comme Oman, le Qatar, l'Arabie Saoudite et les Émirats Arabes Unis.

\section{Un aperçu sur le sommet du classement}

$\mathrm{Au}$ sommet du classement PIRLS2011, les pays les plus performants en lecture sont le Hong Kong, la Russie, la Finlande et Singapour avec des scores très serrés, suivis d'un autre peloton constitué de l'Irlande du Nord, des États-Unis d'Amérique, du Danemark, de la Croatie et du Taipei chinois.

L'Asie de l'Est entretient son leadership du monde dans la réussite scolaire en mathématiques, et ainsi Singapour, la Corée et le Hong Kong, en plus de Taipei chinois et le Japon ont été les plus performants au niveau de la quatrième année primaire. La même tendance est observée au niveau de la deuxième année du collège où se sont respectivement la Corée, Singapour et le Taipei chinois, le Hong Kong et le Japon qui occupent les places du classement.

La Corée du sud et Singapour ont démontré leur leadership dans le domaine de la réussite scolaire en sciences au niveau de la quatrième année primaire, suivis par un groupe formé de la Finlande, du Japon, de la Russie et du Taipei chinois. Quant au niveau de la $2^{\text {ème }}$ année du collège le peloton de tête est conduit par le Singapour suivi respectivement du Taipei chinois, la Corée, le Japon et la Finlande.

Il est également utile de savoir qu'en lecture ( $4^{\text {ème }}$ année du primaire) 33 pays ont réalisé des scores au-dessus de la moyenne internationale alors que 12 n'ont pas pu atteindre ce seuil. De même en mathématiques ( $4^{\text {ème }}$ année du primaire), ils étaient 27 contre 23 , en $2^{\text {ème }}$ année du collège 14 contre 28 . Alors qu'en sciences ( $4^{\text {ème }}$ année du primaire), ils étaient 29 contre 20 , en $2^{\text {ème }}$ année du collège 18 contre 24 .

Notons enfin, c'est important, que la participation des différents pays à ces enquêtes est volontaire, ce qui signifierait que les responsables du SEF sont conscients de l'importance de ces enquêtes et les précieuses données qu'elles mettent à leur disposition. Ajoutons à cela que nul ne peut ignorer, que la base de la bonne gouvernance d'un système éducatif, en particulier, repose sur la disponibilité d'indicateurs élaborés dans une logique systémique, qui n'omet aucun des facteurs intervenant, de quelque manière que ce soit dans la performance des apprentissages. Ceci dit, et mettant à part la non réactivité officielle vis-àvis de rapports PIRLS et TIMSS, les responsables nationaux ont le devoir d'être assez lucides quant aux approches permettant d'entamer la correction des dysfonctionnements et l'amélioration des pratiques. Si la participation à ces enquêtes équivaut à la mobilisation de ressources financières, logistiques et Humaines, leurs résultats, analyses et comparaisons doivent être exploités profondément, si non .... 
Pour conclure, notons qu'après 57 années d'indépendance, Notre système d'Éducation et de Formation :

- n'a pas su se frayer le chemin vers le club des pays dont le système éducatif joue le moteur de développement humain, économique et technologique ;

- ne joue plus son rôle d'ascenseur social, garantissant par là même le principe de l'équité sociale. En effet on peut facilement observer les "systèmes" parallèles que représentent l'enseignement publique et l'enseignement privé sous toutes ses formes.

- ses maux et ses mauvais résultats ne s'expliquent pas par le seul manque de moyens matériels. 